\title{
Role of Cis, Trans, and Inbreeding Effects on Meiotic Recombination in Saccharomyces cerevisiae
}

\author{
Xavier Raffoux,* Mickael Bourge, ${ }^{\dagger}$ Fabrice Dumas,* Olivier C. Martin, ${ }^{*}$ and Matthieu Falque*,1 \\ *Génétique Quantitative et Evolution-Le Moulon, Institut National de la Recherche Agronomique, Centre National de la Recherche \\ Scientifique, AgroParisTech, University of Paris-Sud, Université Paris-Saclay, 91190 Gif-sur-Yvette, France and ${ }^{\dagger}$ Cytometry/Electronic \\ Microscopy/Light Microcopy Facility, Imagerie-Gif, Institute for Integrative Biology of the Cell (I2BC), Commissariat à I'Energie \\ Atomique, Centre National de la Recherche Scientifique, University of Paris-Sud, Université Paris-Saclay, 91198 Gif-sur- \\ Yvette, France \\ ORCID IDs: 0000-0003-0518-0904 (X.R.); 0000-0002-5295-5963 (O.C.M.); 0000-0002-6444-858X (M.F.)
}

\begin{abstract}
Meiotic recombination is a major driver of genome evolution by creating new genetic combinations. To probe the factors driving variability of meiotic recombination, we used a high-throughput method to measure recombination rates in hybrids between SK1 and a total of 26 Saccharomyces cerevisiae strains from different geographic origins and habitats. Fourteen intervals were monitored for each strain, covering chromosomes VI and XI entirely, and part of chromosome I. We found an average number of crossovers per chromosome ranging between 1.0 and 9.5 across strains ("domesticated" or not), which is higher than the average between 0.5 and 1.5 found in most organisms. In the different intervals analyzed, recombination showed up to ninefold variation across strains but global recombination landscapes along chromosomes varied less. We also built an incomplete diallel experiment to measure recombination rates in one region of chromosome XI in 10 different crosses involving five parental strains. Our overall results indicate that recombination rate is increasingly positively correlated with sequence similarity between homologs (i) in DNA doublestrand-break-rich regions within intervals, (ii) in entire intervals, and (iii) at the whole genome scale. Therefore, these correlations cannot be explained by cis effects only. We also estimated that cis and trans effects explained 38 and $17 \%$, respectively, of the variance of recombination rate. In addition, by using a quantitative genetics analysis, we identified an inbreeding effect that reduces recombination rate in homozygous genotypes, while other interaction effects (specific combining ability) or additive effects (general combining ability) are found to be weak. Finally, we measured significant crossover interference in some strains, and interference intensity was positively correlated with crossover number.
\end{abstract}

KEYWORDS meiosis; crossovers; heterozygosity; interference; diversity

$\mathrm{N}$ sexually reproducing organisms, meiosis is a particular type of cell division producing gametes that contain half of the somatic genetic material. Meiotic recombination is a major driver of genome dynamics and evolution because it generates new allelic combinations that can be subject to natural selection. The number of crossover (CO) events and their positions along the chromosomes are tightly regulated, but the mechanisms involved are still not well understood. Obtaining more

Copyright (C) 2018 by the Genetics Society of America doi: https://doi.org/10.1534/genetics.118.301644

Manuscript received July 18, 2018; accepted for publication October 2, 2018; published Early Online October 5, 2018.

Supplemental material available at Figshare: https://doi.org/10.25386/genetics. 7166009.

${ }^{1}$ Corresponding author: Génétique Quantitative et Evolution-Le Moulon, Université Paris-Saclay, Ferme du Moulon, 91190 Gif-sur-Yvette, France. E-mail: matthieu. falque@inra.fr insights into the regulation of recombination rate and $\mathrm{CO}$ distribution would be beneficial for many fields of fundamental and applied genetics, in particular to improve the efficiency of plant breeding (Li et al. 2016). Meiotic recombination starts by programmed DNA double-strand breaks (DSBs) throughout the genome. DSB repair occurs using the homologous chromosome as template, which in turn allows recognition and pairing of the homologous chromosomes (Zickler and Kleckner 2016). DSB repair is achieved by different pathways (Mercier et al. 2015), leading to either COs, which are reciprocal exchanges of genetic material, or noncrossovers (NCOs), which produce gene conversions resulting in a 3:1 allele segregation within a short sequence tract near the break. Sequence divergence between homologous chromosomes can lead to heteroduplex rejection which prevents the formation of double Holliday junctions. In Saccharomyces 
cerevisiae, DNA heteroduplex recognition is achieved by a complex composed of Msh2, the Sgs1 helicase, and the Top3-Rmi1 topoisomerase [Hunter et al. 1996; Chakraborty et al. 2016; see review in Tham et al. (2016)]. In such cases, DSBs can be processed through different pathways (e.g., SDSA), leading to gene conversions but not COs (Alani et al. 1994; Chen and Jinks-Robertson 1999; Chakraborty and Alani 2016). In most organisms, the distribution of DSBs and COs is not homogeneous along chromosomes. At a fine scale (a few kilobases), they are clustered in regions called hotspots, as has been shown for instance in S. cerevisiae (Gerton et al. 2000; Pan et al. 2011; Illingworth et al. 2013), in humans (60\% of COs lying in such hotspots; Coop et al. 2008), and in Arabidopsis thaliana (Drouaud et al. 2013). In S. cerevisiae, 84\% of CO hotspots overlap with gene promoters (Mancera et al. 2008). At the chromosome scale, large "hot" regions showing high CO rates alternate with colder regions (de Massy 2003). The pericentromeric regions of the chromosomes are "cold" in most organisms, e.g., in S. cerevisiae (Mancera et al. 2008), A, thaliana (Giraut et al. 2011), maize (Bauer et al. 2013), and tomato (Anderson et al. 2014). DSBs occur usually in open chromatin regions. In human and mice, many DSB hotspots occur in DNA sequences targeted by the histone H3K4 methyltransferase PRDM9 (Baudat et al. 2009). In S. cerevisiae, the SET1 complex deposits histone H3K4 methylation at the positions of future DSB regions, where the Spp1 protein (Acquaviva et al. 2012) makes a link between H3K4me3 and Spo11, which in turn generates DSBs (Keeney et al. 1997). In maize and $A$. thaliana, CO-rich regions are correlated with low DNA methylation (Mirouze et al. 2012; N. Yelina et al. 2015) and low transposable elements content (Liu et al. 2009; Yelina et al. 2012). In S. cerevisiae, DSB hotspots (which do not directly relate to $\mathrm{CO}$ patterns) colocalize with transcriptionally active regions, especially promoters (Gerton et al. 2000; Borde and de Massy 2013). Also in S. cerevisiae, 40\% of DSBs are repaired to form COs, with the other DSBs being repaired as NCOs or using the sister chromatid as template (Hunter 2015; Marsolier-Kergoat et al. 2018). The ratio between CO and DSB numbers can be regulated at two levels during DSB repair: by driving repair to the homologous chromosomes $v s$. the sister chromatids, and by using the repair pathway leading to the formation of COs vs. NCOs. (Martini et al. 2006, 2011; Matos et al. 2011; Serrentino et al. 2013). CO numbers vary at both inter- and intraspecies levels. However, for $76 \%$ of the species studied (fungi, animals, and plants), the number of COs per bivalent ranges from one to three [see review in Mercier et al. (2015)]. This low variation in CO numbers across species suggests selective constraints keeping recombination levels within a certain range. The presence of at least one $\mathrm{CO}$ per homologous pair can be explained by the need to ensure correct chromosome segregation during the first meiotic division. Concerning the upper limit, possible selective pressures might prevent too many DSBs from becoming COs (Mercier et al. 2015). But this hypothesis remains speculative, especially because some species such as $S$. cerevisiae and $S$. pombe can produce 10 or more COs per bivalent. Furthermore, it was recently shown that in A. thaliana the number of COs can be increased about eightfold without perturbing chromosome segregation (Fernandes et al. 2018). CO numbers also vary at the intraspecific level (McMullen et al. 2009; Cubillos et al. 2011; Bauer et al. 2013; Hunter et al. 2016), although this variation is generally smaller than between species. In $S$. cerevisiae, using four parental strains (Cubillos et al. 2011), it was observed that (1) CO hotspots as well as cold spots are highly conserved among crosses, (2) the number of COs per meiosis varies from 48 to 64.5 , and (3) the recombination rate varies up to $60 \%$ between strains in some intervals. Relatively few studies have investigated the variation of meiotic recombination rate at a broad level within one species. In this work, we characterized the intraspecific diversity of recombination rate in a large part of the $S$. cerevisiae genome. To do so, we developed a highthroughput method to measure CO rates (Raffoux et al. 2018), and we used it to analyze diploids obtained by crossing a SK1 strain to 26 strains taken from a core collection of $S$. cerevisiae strains (see list in Supplemental Material, Table S1; phylogenomic details in Figure S1; Liti et al. 2009). To measure recombination, each strain of the core collection was crossed with eight SK1 testers carrying three different fluorescence markers (mCherry, yECerulean, and Venus, hereafter respectively denoted RFP, CFP, and YFP) at different chromosomal locations (see Materials and Methods). In the resulting diploids, we measured the recombination rate and $\mathrm{CO}$ interference based on 14 genomic intervals covering chromosomes VI and $\mathrm{XI}$, and part of chromosome I. Our results show up to 2.5-fold differences in recombination rate when considering all pooled intervals and up to ninefold differences in some intervals. Our data set indicates also a clear positive correlation between $\mathrm{CO}$ numbers and genome-wide sequence similarity between homologs in the hybrids, and thus a negative correlation between recombination and observed heterozygosity. However, concomitantly, the correlation was weaker when using sequence similarity within the interval where recombination is measured. To obtain further insights, five strains were intercrossed in an incomplete diallel design (among the 15 possible parental combinations, only 10 crosses produced diploids able to sporulate). The recombination rate of these 10 diploids was then analyzed in one interval of chromosome XI. Altogether, we find that sequence similarity between homologs (and thus heterozygosity) plays a major role in the observed variation of recombination rate, and that complete homozygosity lowers recombination, a phenomenon that can be thought of as an inbreeding depression.

\section{Materials and Methods}

\section{Biological material}

The collection of $26 S$. cerevisiae strains used in this study comes from the Saccharomyces Genome Resequencing Project (Cubillos et al. 2009; Liti et al. 2009), and strains were kindly provided by F. Cubillos (Universidad de Santiago de Chile, Santiago, Chile). These strains were collected from 
various geographical areas and types of habitats (Figure S1 and Table S1). The eight SK1 tri-fluorescent testers strains (SK1-I-R2C3Y4, SK1-VI-C1Y2R3, SK1-VI-R3Y4C5, SK1-XIR1C2Y3, SK1-XI-Y3R4C5, SK1-XI-R4C5Y6, SK1-XI-Y6C7R8, and SK1-XI-R8Y9C10, hereafter referred to as "testers") used to measure recombination are described in a previous paper (Raffoux et al. 2018). Each of them contains three reporter genes distant by $\sim 30 \mathrm{cM}$ on a same chromosome, coding for three different fluorescent proteins that can be detected in flow cytometry. That nice feature allowed us to use trifluorescent testers rather than bi-fluorescent ones, speeding up the process of measuring recombination rates; as a bonus, we also obtained measures of genetic interference since we were able to detect the presence of double recombinants.

\section{Sporulation efficiency}

Each of the 26 MAT a strains of the collection was crossed with the MAT $\alpha$ tester SK1-XI-R1C2Y3 to produce a hybrid diploid and spores as described by Raffoux et al. (2018). At days 1, 2, $3,4,7,8,9,10,11$ of incubation on solid sporulation medium (2.5\% yeast extract, $1 \%$ glucose, $10 \%$ potassium acetate; sporulation in this work was always induced on solid medium for practical reasons related to the number of samples to be processed at the same time in the incubator) at $30^{\circ}$, and cells were picked up and resuspended in $10 \mu \mathrm{l} \mathrm{H}_{2} \mathrm{O}$ on a microscope slide. Tetrads and vegetative cells were counted at $\times 1000$ magnification. To assess sporulation efficiency, only small spots were sampled on the surface of the sporulation medium, which may have caused heterogeneity leading to some sporulation kinetics curves showing decreasing regions (e.g., YS9 between days 3 and 4). Thus, for recombination analyses, very large areas were scraped to avoid such heterogeneity, and reproducibility was indeed high.

\section{Spore viability}

At day 10 of the sporulation efficiency experiment, we scraped one quadrant of each of the 26 petri dishes and prepared spores as described by Raffoux et al. (2018) for FACS sorting. We selected events corresponding to the size of spores using a gate in the side scatter (SSC)-height-log $v s$. forward scatterheight-log graph (Summit software; Beckman Coulter, Brea, $\mathrm{CA}$ ), then we discarded events containing more than one cell using a gate in the SSC-height-log vs. SSC-area-log graph [see Materials and Methods in Raffoux et al. (2018)]. One spore per well was distributed in two 96-well plates containing $100 \mu \mathrm{l}$ solid YPD medium. After $48 \mathrm{hr}$ incubation at $30^{\circ}$, we counted the number of wells in which a colony had grown. In rare cases, two colonies were observed in the same well and these events were discarded from further analyses. Thus, 192 spores were analyzed per condition.

\section{Recombination rate and interference measurements on the collection}

As it was technically impossible to handle all strains and all testers in the same experiment, we worked with each tester, one at a time. Thus, for one given experiment, each of the
26 MAT a strains of the collection was deposited with one given MAT $\alpha$ tri-fluorescent tester strain on solid YPD medium and incubated one night at $30^{\circ}$ to produce diploid cells. Cells were then transferred to sporulation medium. To capture possible variation due to environmental heterogeneity, the experiment was designed in the following manner: (1) for each cross, four petri dishes were placed at different positions in the incubator to provide four replicates, and (2) in each experiments, the control (Y12 MAT a) $\times(\mathrm{SK} 1-\mathrm{VI}-$ Y3R4C5 MAT $\alpha$ ) diploid was added. After 10 days at $30^{\circ}$, tetrads were picked up by scraping one-quarter of the petri dish surface, and spores were then isolated as described by Raffoux et al. (2018). The spore suspensions were analyzed with a MoFlo ASTRIOS flow cytometer (Beckman Coulter) and the associated software Summit. Vegetative cells were filtered out based on SSC and forward scatter as described above, and then the fluorescence intensity was analyzed for each spore in the mCherry, yECerulean, and Venus channels (excitation at 561, 405, and $488 \mathrm{~nm}$, respectively; emission at $614 / 20,448 / 59$, and 526/52 nm, respectively). To quantify recombination rates and coefficients of coincidence (CoC), we used the mathematical model given in Raffoux et al. (2018) to take into account the fact that fluorescence can be extinguished at a low rate (see Figure S2 and File S1). As recombination rate values of the $(Y 12$ MAT a) $\times($ SK1-VIY3R4C5 MAT $\alpha$ ) control sample did not show significant variation between the eight experiments corresponding to the eight testers (ANOVA $P$-value $=0.99$ ), results were normalized using this control as a standard (see File S1). The CoC for a pair of intervals is defined as the ratio between the experimental frequency of double recombinants and its theoretical frequency in the absence of interference. Absence of interference means that recombination events in the two intervals are independent, and thus the theoretical frequency of double recombinants is simply the product of each interval's recombination fraction. Since CoC values strongly depend on recombination rate in the two intervals, we cannot compare CoC values across different strains or testers. We thus used a simulation approach to map the correspondence between CoC and the parameter $\nu$ of the Gamma model (McPeek and Speed 1995) for each strain/tester combination. First, it was necessary to simulate the relationship between recombination fraction and number of COs for each value of $\nu$ (see examples in Figure S3), and then the relationship between CoC and $\nu$ (see examples in Figure S4; see details in File S1). The parameter $\nu$ is a quantitative measurement of interference strength and its value is 1 in the absence of interference, $>1$ in the presence of positive interference, and $<1$ in the presence of negative interference. In the Gamma model framework, this parameter does not depend on recombination rate and thus its values may be compared across strains and testers.

\section{Score of sequence similarity at different scales}

Reference sequences of all strains studied come from the Saccharomyces Genome Resequencing Project (Cubillos 
et al. 2009; Liti et al. 2009). The sequence similarity percentage between homologous genomes was calculated at different scales: (1) genome-wide, (2) in the whole chromosome carrying the considered markers, (3) in the interval surrounded by the two markers analyzed, (4) within that interval but focusing only on the DSBs-rich regions defined as 300 bp regions for which Pan et al. found at least 100 Spo11-associated oligo reads (Pan et al. 2011), and (5) in the $30 \mathrm{~kb}$ regions surrounding the interval. Similarity percentages were calculated both ways, using the SK1 sequence as query blasted against the other parent as subject, and the reciprocal analysis using the SK1 sequence as subject and the other parent as query. Motivated by what occurs during the repair of meiotic DSBs, for each pair of sequences considered, the query sequence was sliced in 200 bp windows sliding with a $50 \mathrm{bp}$ step. Only windows that did not contain any " $N$ " in their sequence (92.6\% of the cases; SD 7.9\%) were considered. For each window, we calculated the sequence similarity percentage as the fraction of identical nucleotides in the first high-scoring-segment pair multiplied by its length and divided by the size of the window (200), and multiplied by 100 . We then took the average percentage of similarity for all windows within the region considered, calculated both ways. These computations were carried out using R scripts calling standalone BLAST + (Camacho et al. 2009). Blast was preferred to sequence alignment software because it is much quicker and complete alignments were not necessary here.

\section{Data availability statement}

The authors affirm that all data necessary for confirming the conclusions of this article are represented fully within the article and its tables and figures. File S1 contains additional explanations on the methods used. Supplemental material available at Figshare: https://doi.org/10.25386/genetics. 7166009.

\section{Results}

\section{Sporulation, spore viability, and recombination rate}

Because of the large genetic diversity explored in this work, we first assessed the correct progress of meiosis using sporulation rate and spore viability as proxies. When crossing all strains of the collection (see Materials and Methods) with SK1, sporulation rates reached a plateau before 10 days on the sporulation medium (Figure S5), and the values of the plateau ranged from 14 to $85 \%$ across hybrids with a continuous variation, the maximum being reached for the SK1 $\times$ SK1 diploid, which is completely homozygous (Figure S6A). Sporulation rates and spores viabilities obtained in this work using a simple solid medium (for practical reasons) were on average lower than those usually obtained with optimized conditions. Spore viability ranged from 1.5 to $85 \%$, with the hybrids from strains UWOPS03_461_4, UWOPS05_217_3, UWOPS05_227_2 (Malaysian wild strains), and YS9 (Asian baking strain) producing almost no viable spores (Figure
S6B). Such low viability may denote abnormalities in the meiotic process, probably associated with chromosomal rearrangements previously described for the Malaysian clade (Dujon and Louis 2017). Therefore we discarded these four strains. Spore viability was not correlated with the sporulation rate $(P$-value $=0.16)$, indicating that these two biological processes are relatively independent. Finally, the average recombination rate over the eight testers was significantly positively correlated with spore viability $\left(r^{2}=0.49 ; P\right.$-value $=$ $\left.7.2 \times 10^{-5}\right)$.

\section{Wide diversity of recombination rate in the collection}

When pooling the information obtained from all intervals of the eight testers, we obtained global recombination rates ranging from 0.20 to $0.51 \mathrm{cM} / \mathrm{kbp}$ across the 22 hybrids tested. The highest value corresponds to the SK1 $\times$ SK1 diploid (Figure 1). Recombination rates averaged over hybrids varied significantly between chromosome I (0.47 cM/kbp), chromosome VI (0.39 cM/kbp), and chromosome XI (0.30 $\mathrm{cM} / \mathrm{kbp}$ ) (Tukey's Honest Significant Difference test $P$-value $<10^{-7}$ ), and between individual testers as well as between individual intervals delimited by fluorescent markers (Figure 2 and Table S2; see each hybrid in Figure S7). The patterns of recombination rate along chromosomes were significantly different between hybrids for some intervals, but all hybrids showed the same decreasing recombination rate tendency in the vicinity of centromere regions except for chromosome I, for which there is a strong DSB hotspot in the interval containing the centromere (Figure 2; see each hybrid in Figure S7). For each interval, the ratio between the most and least recombining hybrids ranged from 1.8 to 9.5 . Note that the SK1 $\times$ SK1 diploid had the highest recombination rate only for intervals 2 and 10. Analyses of variance revealed significant effects of hybrids, intervals, and hybrid $\times$ interval interactions on recombination rate $\left(P\right.$-value $<2.2 \times 10^{-16}$ for each effect). Further, we observed a significant effect of the geographic origin on the global (eight testers pooled) recombination rate of the hybrid (ANOVA $P$-value $=0.009$ ). Specifically, pairwise significant differences were observed between hybrids involving strains collected in Africa and America (Tukey's HSD test $P$-value $=0.049$ ). Genome-wide sequence-based phylogenetic groups (Liti et al. 2009; see Figure S1) also showed a significant association with recombination rate (ANOVA $P$-value $=3.9 \times 10^{-6}$ ). Specifically, pairwise significant differences were observed between the West African phylogenetic group and all other groups (Tukey's HSD test $P$-values $<10^{-4}$; see Figure S8). However, West African strains gave almost the highest recombination rates of the collection for eight intervals, but almost the lowest for four intervals, and are ranked in the middle for the remaining two intervals (Figure S9), pointing to very different behaviors between intervals. Because adaptation to a changing environment can drive evolution toward higher recombination rate (Aggarwal et al. 2015), we analyzed hybrids of strains grown in laboratory, clinical, or fermentation habitats (supposed to be stable environments). Surprisingly, 


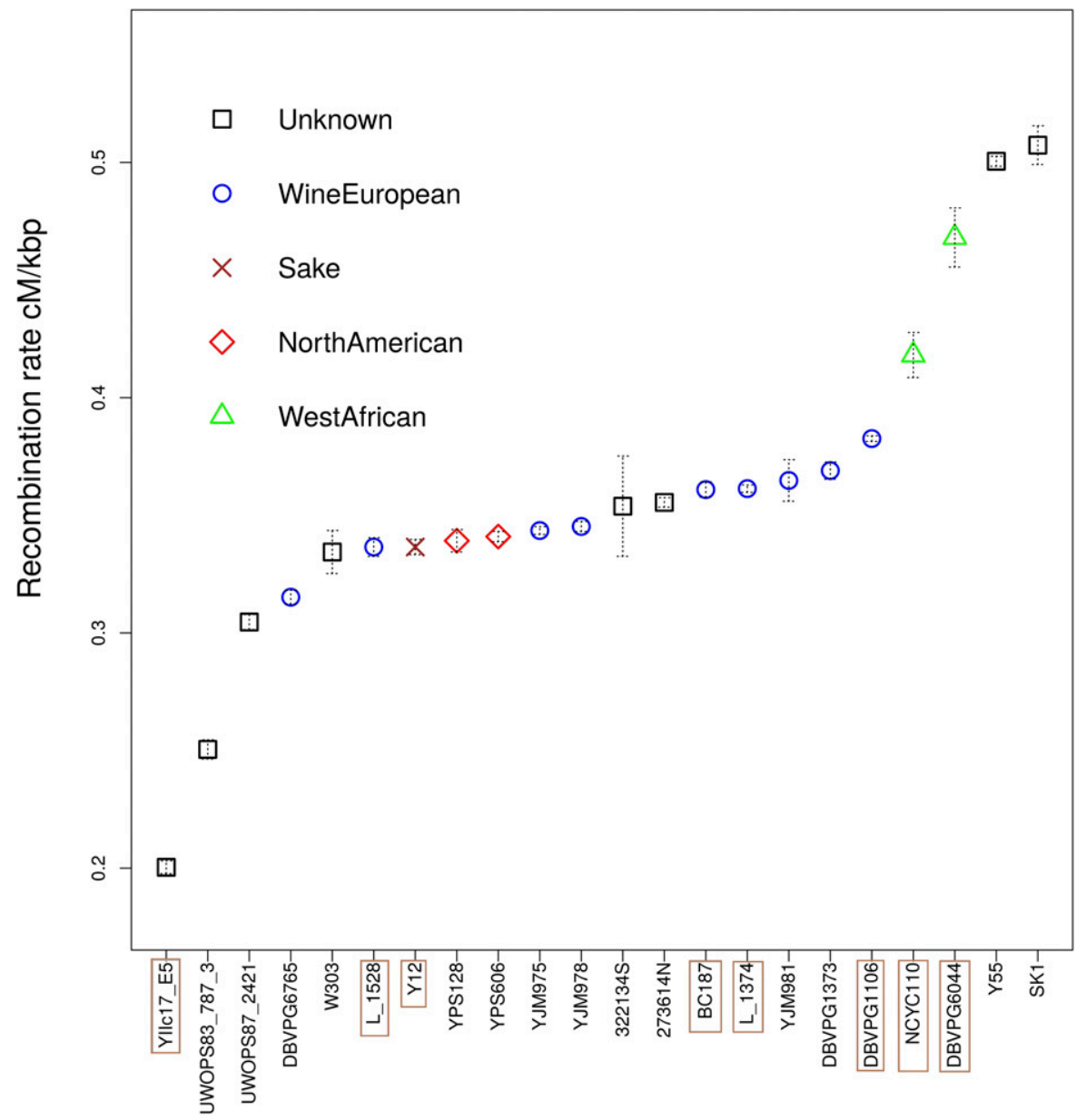

Figure 1 Average recombination rate over the 14 intervals for each strain of the collection crossed to SK1 testers. Strain names bordered in brown indicate domesticated strains. Symbols and colors refer to the phylogenetic group of the strains. Error bars indicate 95\% confidence intervals based on four biological replicates.

they had significantly higher recombination rates than hybrids of strains coming from wild habitats which may correspond to more changing environments (Tukey's HSD test $P$-values $<10^{-4}$; see Figure S8).

\section{Relative importance of cis and trans effects on recombination}

Cis effects generally refer to situations where DNA sequence variation in a given region controls a phenomenon (e.g., gene expression or recombination) arising in that particular region or in its neighborhood. On the contrary, trans effects act remotely via diffusing factors. To quantitatively estimate and test the respective roles of cis and trans effects in our recombination data, we formalized the question by defining trans effects as mechanisms modulating recombination by the same extent in all intervals studied for one given hybrid. Given this definition, trans effects correspond to the general effect of the hybrid on recombination (excluding interaction with intervals), and cis effects correspond to the specific effect of hybrid $x$ interval interactions on recombination. We observed highly significant effects of hybrid, interval, and hybrid $\times$ interval interaction on recombination rate (ANOVA $P$-value $<10^{-15}$ for all three effects, the model explaining $99.4 \%$ of the total phenotypic variance because there was hardly any variation across replicates). This approach allowed us to estimate that cis effect explained $38.3 \%$ of the variance of recombination rate and that trans effects explained $17.0 \%$ of that variance.

\section{Relationship between recombination rate and DSB levels}

For most hybrids, recombination rates and DSB patterns showed a positive correlation except in the region between markers $\mathrm{Y} 2$ and $\mathrm{R} 3$ of chromosome VI, and in the region between Y9 and C10 of chromosome XI (Figure 2; see also each hybrid in Figure S7). Patterns of recombination rate along chromosomes and average SK1 DSB levels [data from Pan et al. (2011)] were both low near the centromere, except for chromosome I (Figure 2).

\section{High levels of heterozygosity reduce recombination}

To investigate the correlation of recombination rate with sequence similarity between homologous chromosomes (which is 1 - the observed heterozygosity) across the different hybrids, we considered successively five scales of sequence similarity: the pool of all intervals studied, the pool of all intervals on each chromosome, each interval separately, DSB-rich regions within each interval, and $30 \mathrm{~kb}$ regions surrounding each interval (see Materials and Methods). We found a significant positive correlation between average 


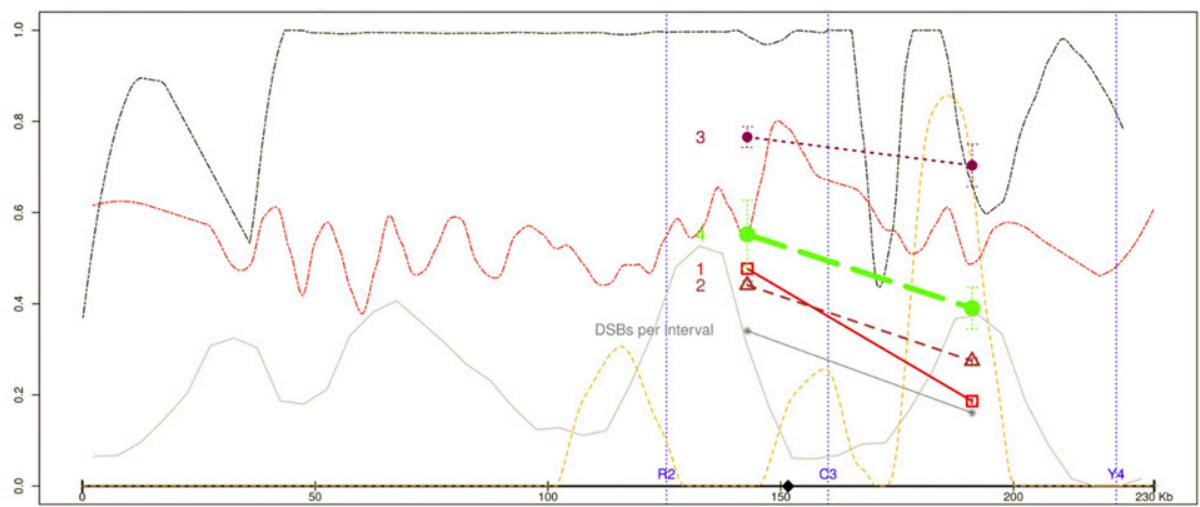

Physical position on chromosome I

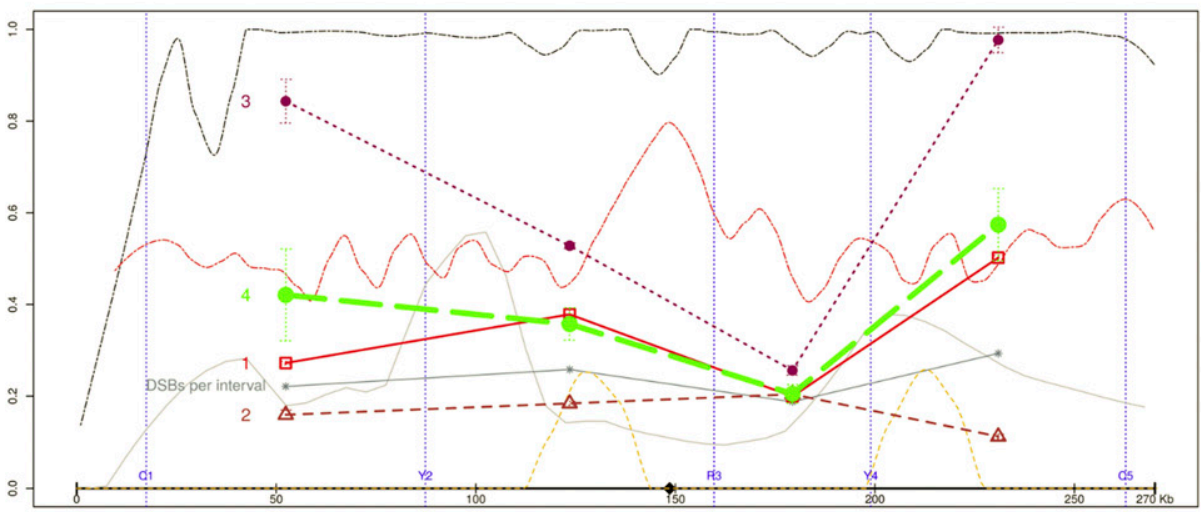

Physical position on chromosome VI

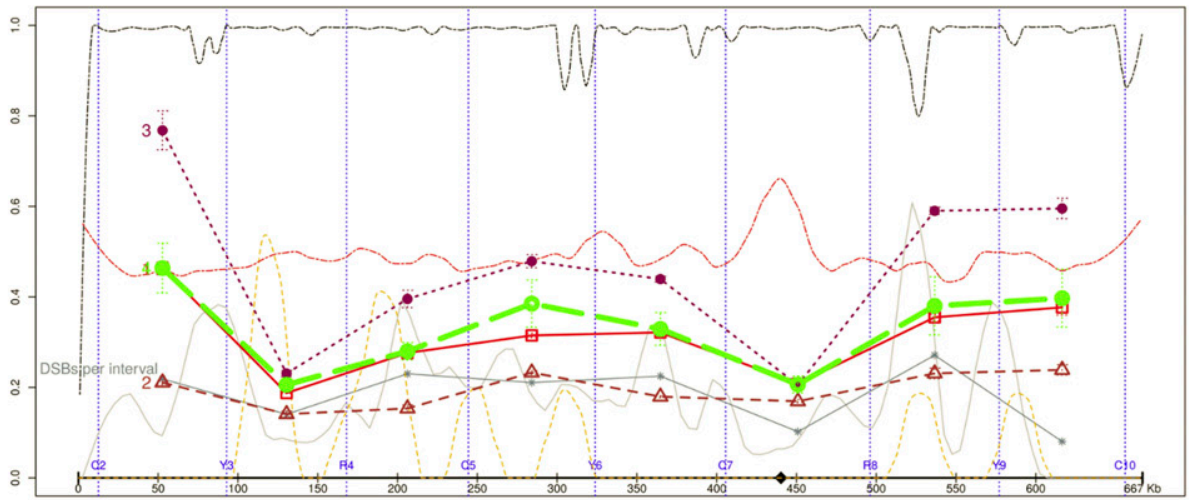

Physical position on chromosome XI
Figure 2 Recombination rates in centimorgan per kilobase pair along chromosomes for hybrids between SK1 and strains UWOPS87_2421, YllC17_E5, and SK1, and for the mean of the 22 hybrids (dashed green line). Error bars indicate $95 \%$ confidence intervals based on four biological replicates. $x$-axis: physical position along chromosome I (top panel), VI (middle panel), or XI (bottom panel). Also shown are DSBs per interval: average frequency of doublestrand breaks per base (Pan et al. 2011) between markers; DSB level: average number of DSBs per $5 \mathrm{~kb}$ window smoothed using "loess;" H3K4Me3 level: arbitrary units based on Liu et al. (2005) and smoothed using "loess;" Rec 8 level: arbitrary units based on Sun et al. (2015) and smoothed using "loess;" and sequence similarity: proportion of identical bases between homologs (see Materials and Methods) smoothed using "loess." Vertical blue dashed lines indicate the positions of fluorescent markers. Diamonds at the bottom show centromere positions.

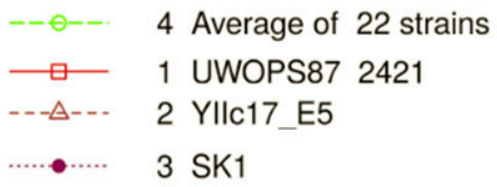

recombination rate and sequence similarity when pooling all intervals $\left(r^{2}=0.43 ; P\right.$-value $\left.=9 \times 10^{-4}\right)$ (Figure 3$)$, as well as when pooling intervals for each chromosome $\left(r^{2}>0.2 ; P\right.$ value $<0.04$ ) (Figure S10). The three chromosomes investigated thus seem to have similar correlations. When considering the 14 intervals separately, we found significant
DSBs per interval

DSB level

H3K4me3 level

Seauence similarity

Rec8 level positive correlations between sequence similarity and recombination rate for nine of them. Analysis of sequences flanking these 14 intervals on both sides showed that only five intervals gave significant positive correlations (Table S3). Finally, focusing on sequence similarity within DSB-rich regions in these 14 intervals, nine intervals showed significant positive 


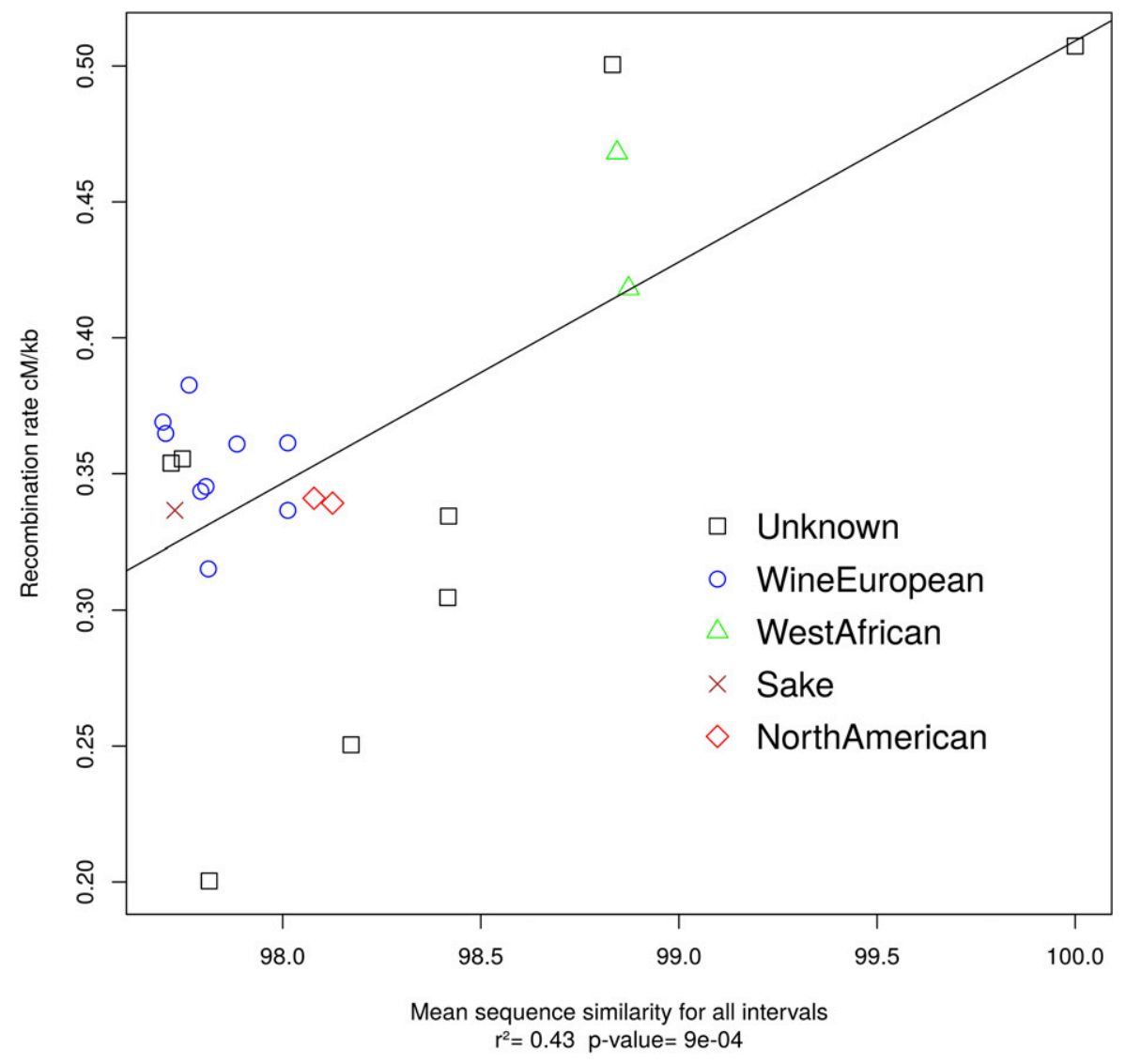

Figure 3 Correlation between sequence similarity when pooling all intervals and the mean recombination rate of hybrids. $X$-axis: score of sequence similarity (see Materials and Methods), $y$-axis: for each strain of the collection, average of the recombination rates obtained in the 14 intervals. Colors indicates the phylogenetic group of the strains. correlations (Table S3). This correlation is expected because sequence divergence greater than $\sim 1 \%$ is known to lead to heteroduplex rejection by the mismatch repair machinery, and recombination suppression (Chen and Jinks-Robertson 1999). Elsewhere, the correlation between recombination rate and sequence similarity in DSBs rich regions is weaker than when considering the whole sequence spanned by intervals. This suggests that in our data, CO number is not only controlled by local sequence similarity at the sites of DSBs repair. Similarly, the correlation between recombination rate and sequence similarity within the interval studied is weaker than when considering genome wide sequence similarity, which again points to the existence of trans effects in addition to cis effects for controlling CO number.

\section{CO interference analysis}

To quantitatively compare interference strength across strains and chromosomal regions, we used the $\nu$ parameter of the Gamma model (McPeek and Speed 1995), inferred for each pair of adjacent intervals (corresponding to one tester) from its $\mathrm{CoC}$ and its two recombination fractions measured for a strain $\times$ tester combination (see Materials and Methods and supplemental methods in File S1). As in our previous study (Raffoux et al. 2018), we discarded the tester SK1-XI-R1C2Y3 from interference analyses because its first interval is anomalously small ( $5557 \mathrm{bp}$ ). When pooling the information given by the seven testers, we obtained $\nu$ values ranging from 0.54 to 1.53 across hybrids (Figure 4; see also CoCs in Figure S11). Most hybrids show either no interference $(\nu \approx 1)$ or positive interference $(\nu>1)$. However, the two strains YIIc17_E5 and UWOPS83_787_3, which also have the lowest genome-wide recombination rates, display negative interference $(\nu<1)$. Interference patterns along chromosomes were also significantly different between some hybrids (Figure S12). We found significant effects of hybrid, tester, and interaction hybrid $\times$ tester on interference strength (ANOVA $P$-value $<$ $2.2 \times 10^{-16}$ ). Interference strength and average recombination rate were positively correlated across the seven testers (Figure 5), even when discarding the two outlier strains YIIc17_E5 and UWOPS83_787_3 from the data $\left(r^{2}=0.56\right.$; $P$-value $=10^{-4}$ ).

\section{Inbreeding reduces recombination}

To obtain further insights on the control of recombination rate, we measured the genetic length of interval Y9C10 on chromosome XI for 10 hybrids obtained by crossing five parental strains in an incomplete diallel experiment (see Figure 6). As above, the recombination rates in this diallel experiment showed a significant correlation with sequence similarity between homologs $\left(P\right.$-value $=7 \times 10^{-10} ; r^{2}=$ 0.4). Recombination rate is a quantitative trait displaying genetic diversity (Figure 1). As such, it may be controlled by several types of mechanisms involving QTLs possibly interacting with each other. These QTL may have two kinds of 


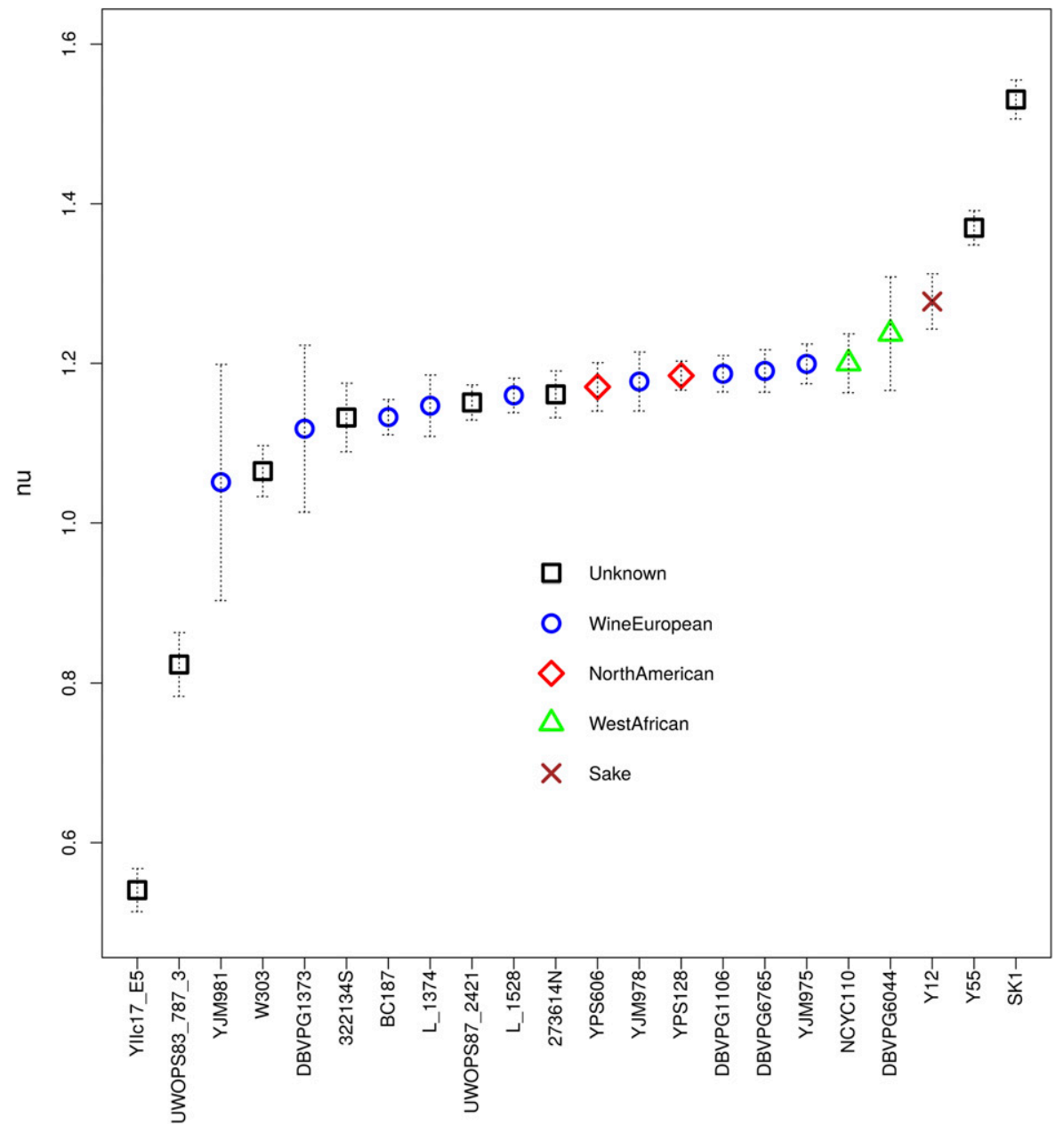

Figure 4 Diversity of interference strength measured by the $v(n u)$ parameter of the Gamma model across hybrids. $x$-axis: name of the parent crossed with SK1 tester to produce the hybrid. $y$-axis: average $\nu$-value over all testers except SK1-XI-R1C2Y3, which has two very close markers and was not considered for these averages. Error bars indicate 95\% confidence interval based on four biological replicates. Colors refer to the phylogenetic group of the strains. effects: (1) additive effects of individual alleles, which sum up in the hybrid, referred to as general combining ability (GCA; Sprague and Tatum 1942), and (2) interaction effects between alleles either at the same locus (including dominance, overdominance, and inbreeding) or between alleles at different loci (epistasis), referred to as specific combining ability (SCA; Sprague and Tatum 1942). The effect of heterozygosity on recombination rate may be considered as a particular type of SCA because there is no additive effect associated with individual sequences and the recombination rate depends on each pair of homologous sequences. Therefore we used the pairwise sequence similarity as a quantitative explicative variable in our diallel analysis, thereby distinguishing sequence similarity effects from other interaction effects. We then used the hierarchical generalized linear model below, considering sequence similarity between homologs as a fixed effect, and GCA, SCA, and inbreeding as random effects. Specifically, the statistical model sets

$$
Y_{i j k}=\mu+\alpha S_{i j}+\mathrm{GCA}_{i}+\mathrm{GCA}_{j}+\mathrm{SCA}_{i j}+\mathrm{INB}_{i j}+\varepsilon_{i j k},
$$

where $Y_{i j k}$ is genetic distance (in centimorgans), measured in the hybrid formed by crossing strain $i$ and strain $j$ for the replicate $k ; \mu$ is intercept (in centimorgans); $\alpha$ is the coefficient associated with sequence similarity effect (in centimorgans per percent of similarity); $S_{i j}$ is the percentage of sequence similarity between strain $i$ and strain $j$ (see Materials and Methods); $\mathrm{GCA}_{i}$ is the GCA (in centimorgans) of strain $i$; $\mathrm{GCA}_{j}$ is the GCA (in centimorgans) of strain $j$; $\mathrm{SCA}_{i j}$ is the SCA (in centimorgans) of the hybrid obtained by crossing strain $i$ and strain $j$ when $i \neq j$, set to 0 when $i=j$, calculated as $Y_{i j .}-1 / 2\left(Y_{i . .}+Y_{. j .}\right)-\mu$; $\mathrm{INB}_{i j}$ is the inbreeding effect when $i=j$ (in centimorgans), calculated as $Y_{i i}-Y_{i . .}-$ $\mu$; and $\varepsilon_{i j k}$ is the residual variance.

To estimate the parameters, we used the $\mathrm{R}$ hglm package (Rönnegård et al. 2010; Alam et al. 2015) as described by Lenarcic et al. (2012) and Nepoux et al. (2015). This program uses a Bayesian approach to fit hierarchical generalized linear models. We found that the Akaike information criterion decreased when adding factors one after the other, indicating that all parameters of the model are relevant. We further checked that there was a strong significant correlation between experimental and predicted phenotypic values $\left(r^{2}=\right.$ $0.78 ; P$-value $\left.=4.5 \times 10^{-45}\right)$ (Figure S13). The results of the diallel analysis are given in Figure S14. Values of effects are relative to the intercept $\mu$ which would be the phenotypic value obtained if all effects were null. The GCA results showed that strain DBVPG6044 had a significantly higher 


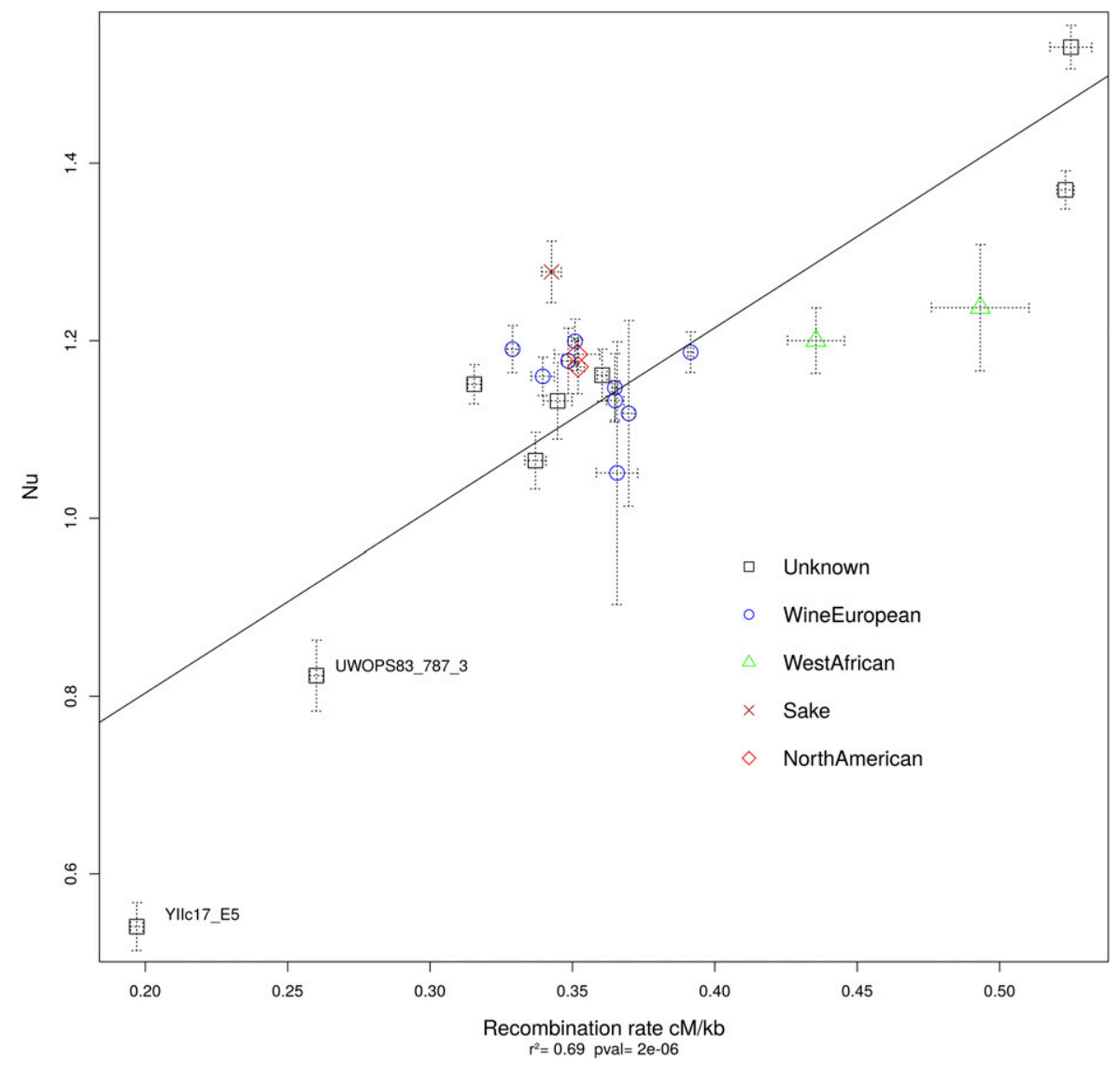

Figure 5 Correlation between average interference intensity $\nu(\mathrm{Nu}$; parameter of the Gamma model), and average recombination rate over all testers except SK1-XI-R1C2Y3, which has two very close markers and was not considered for these averages. Error bars indicate $95 \%$ confidence interval based on four biological replicates. Colors refer to the phylogenetic group of the strains.
GCA value ( $+8.5 \mathrm{cM}$ from the intercept value) than the four other strains (between -3.4 and $0 \mathrm{cM}$ ), which were not very different from each other. SCA results showed some differences between parental combinations (ranging from -3 to $+4.5 \mathrm{cM}$ ) but the effects remained limited. In the cases of SK1 and YPS128, for which we could measure the recombination rates in the homozygous diploids, we observed strong inbreeding effects, in effect depressing the recombination rates in a major way ( $-36.7 \mathrm{cM}$ for SK1 and $-23.3 \mathrm{cM}$ for YPS128). Finally, the estimated effects of sequence similarity $(\alpha S)$ ranged from 0 to $+44.4 \mathrm{cM}$ across hybrids, the two highest values corresponding to homozygous diploids. Thus in our experiment, hybrids from distantly related strains show that heterozygosity decreases recombination rate, but we also see from the two homozygotes having negative inbreeding effects that high levels of homozygosity seem to decrease recombination rate. Altogether, $\alpha S$ and inbreeding effects were much stronger than other effects, suggesting that sequence similarity may be the strongest factor driving the genetic diversity of recombination rate within $S$. cerevisiae strains.

\section{Discussion}

\section{Intraspecific diversity of recombination}

When the level of divergence between homologous chromosomes is too high, DSBs cannot be repaired through the homologous recombination pathway but may be repaired as gene conversions because of heteroduplex rejection by the mismatch repair pathway. This may prevent $\mathrm{CO}$ formation, leading to aneuploidy and loss of spore viability (Chambers et al. 1996; Hunter et al. 1996). In our study, we thus discarded the hybrids showing strong spore viability defects, to keep only those which are relevant for studying homologous recombination. We observed recombination rates (averaged across the eight testers) between 0.20 and $0.51 \mathrm{cM} / \mathrm{kbp}$, which is consistent with previous results in budding yeast genome-wide analyses: $0.4 \mathrm{cM} / \mathrm{kbp}$ (see Figure 2 of Cubillos et al. 2011), $0.61 \mathrm{cM} / \mathrm{kbp}$ (Mancera et al. 2008), or from 0.29 to $0.63 \mathrm{cM} / \mathrm{kbp}$ for chromosome VII left arm (Malkova et al. 2004). We observed a negative correlation (although involving only three chromosomes) between chromosome physical size and recombination rate, in accordance with a mechanism previously proposed in yeast (Kaback et al. 1992). Across our 22 strains, we obtained an average 2.55fold variation of recombination rate that can be compared to the observation by Cubillos et al. (2011) of a fourfold variation between crosses of four genetically distant $S$. cerevisiae strains. This could be compared to the case of maize, where only $30 \%$ variation was measured in genome-wide CO numbers between 23 (Bauer et al. 2013) and 25 (McMullen et al. 2009) hybrids, based on genetic mapping; such far more modest variation might be attributable to less intraspecific divergence in maize than in $S$. cerevisiae. We also observed 


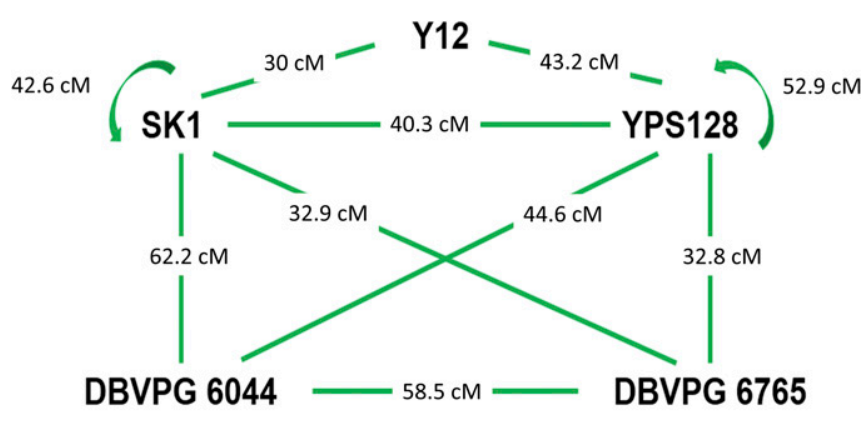

8 inter-crosses and 2 self-crosses Interval XI-Y9C10

Figure 6 Hybrids obtained by crossing five parental strains. Each green line represents a cross and corresponding numbers indicate the genetic distance in centimorgan measured in the interval.

that West African strains gave hybrids with the highest average recombination rates (Figure S8; although the opposite trend was observed in a few intervals, see Figure S9). This is in accordance with our results on the effect of heterozygosity since these strains are phylogenetically the closest to SK1 (Figure S1). The present study also indicates that the recombination landscape along chromosomes is different across strains: the ratio between the most and least recombining hybrids ranged from 1.8 to 9.5 depending on the interval. In maize (McMullen et al. 2009), an average 2.9-fold variation in CO number was reported between 25 hybrids, with some intervals showing up to 30-fold differences. Such high levels of variation of recombination rate across intervals suggest that some determinants affect recombination in nearby locations (cis effects). Finally, we observed significantly different recombination rates depending on the habitat of the parental strains, but there is no indication that strains living in changing environments may have evolved higher recombination rates to adapt more easily, as previously hypothesized (Bürger 1999; Aggarwal et al. 2015; McDonald et al. 2016; Kosheleva and Desai 2017). Hybrids from laboratory strains recombined more than the other groups, but those from strains domesticated for fermentation did not. This gives no clear support to the hypothesis that domesticated strains evolved higher recombination rate (Ross-Ibarra 2004), or were selected indirectly for their higher recombination rate since creating more genetic combinations can play positive roles for adaptation. Note that higher recombination may also have deleterious effects by breaking up established favorable arrangements, although $A$. thaliana mutant implementing nearly eight times more COs did not show any detectable phenotypic defect (Fernandes et al. 2018).

\section{Intraspecific diversity of $\mathrm{CO}$ interference}

Through our measurements of $\mathrm{CoC}$ and interference strength $(\nu)$, we observed positive CO interference for most testers and hybrids, which is in accordance with previous studies reporting interference in $S$. cerevisiae (de los Santos et al. 2003; Malkova et al. 2004; Stahl et al. 2004; Chen et al. 2008).
However, the two hybrids YIIc17 E5 $\times$ SK1 and UWOPS83_787_3 $\times$ SK1 showed negative interference. These two hybrids are among those with the lowest recombination rate, sporulation rate, and spore viability of the collection. In such crosses between distantly related parents, negative interference might be justified a posteriori as being due to meiotic defects. Specifically, if two homologs do not pair in some of the meioses, CO events will be statistically positively correlated because the data will consist of a mixture where some cases have pairings and others do not. In a similar vein, if homologs stochastically pair only along part of their length, COs will be restricted to those paired regions and thus in effect they will be subject to clustering. Both situations result in apparent negative interference even if there is positive interference between COs for each meiosis or paired region. This is in direct analogy with what was observed in the Arabidopsis axr1 mutant (Jahns et al. 2014).

We also observed significant variation of interference across hybrids. To our knowledge, intraspecific diversity of interference strength had never been assessed before in $S$. cerevisiae, but in maize, Bauer et al. (2013) reported significant differences among 23 hybrids based on the Gamma model. We also measured variations of interference intensity along and between chromosomes, as already reported in $S$. cerevisiae (Zhao et al. 1995; Mancera et al. 2008) and in Arabidopsis (Basu-Roy et al. 2013). Our results showed significant positive correlations (averaged across seven testers) between recombination rate and interference strength, whereas in maize, Bauer et al. (2013) reported a significant negative correlation. It is commonly hypothesized that interference reduces $\mathrm{CO}$ number while ensuring the obligatory $\mathrm{CO}$ (Chakraborty et al. 2017). Indeed, there seems to be selective pressure against too many COs, although the reasons are unclear (Mercier et al. 2015). The maize results of Bauer et al. (2013) are in accordance with this hypothesis, whereas our results in yeast are not. A tentative explanation may come from the fact that in maize, each meiocyte undergoes almost 500 DSBs that lead to $\sim 20$ COs (Anderson et al. 2003; Pawlowski et al. 2003), whereas in S. cerevisiae, $40 \%$ of the DSBs lead to the formation of COs (Brown et al. 2013). The $\mathrm{DSB} / \mathrm{CO}$ ratio is then $\sim 25$ in maize to be contrasted with 2.5 in $S$. cerevisiae. So in the context of selective pressure against both too many COs and zero CO, a high number of DSBs together with strong interference could fulfill both constrains. One would then expect many more DSBs than COs, and a negative correlation between interference and recombination, as observed in maize. On the other hand, as the DSB/CO ratio is low in $S$. cerevisiae, it is less plausible there to consider interference as the main mechanism at work to limit CO number, and this might explain the difference between our results in yeast and results in maize (Bauer et al. 2013).

\section{Genetic control of recombination rate}

Effect of heterozygosity and homozygosity on recombination rate: Considering all intervals pooled, the recombination rates in our study showed a significant positive 
correlation with sequence similarity between the two parents of the hybrid (i.e., a negative correlation between heterozygosity and COs). This strong correlation $\left(r^{2}=\right.$ $0.43 ; P$-value $=9 \times 10^{-4}$; Figure 3$)$ is in accordance with previous studies showing that heterozygosity can have an inhibitory effect on homologous recombination in yeast (Borts and Haber 1987) or in A. thaliana (Opperman et al. 2004). In our study, sequence similarity in DSB-rich regions did not explain recombination rate better than sequence similarity in whole intervals, suggesting that the sequence similarity in the region of strand invasion is probably not the main determinant of DSB commitment into CO vs. NCO or repair on the sister chromatid. We used DSBs pattern obtained from a homozygous SK1 strain, whereas our hybrids are heterozygous between SK1 and other strains; nevertheless, this is not a problem because DSB landscape variation has been shown to be very modest between S. cerevisiae strains (Lam and Keeney 2015). In all likelihood, DSBs landscapes are similar between SK1 and our hybrids. Elsewhere our analysis of sequence similarity in regions flanking the 14 intervals on both sides showed a significant positive correlation with the recombination rate within the interval in five cases (four of which also being significant when considering sequence similarity within the intervals). This suggests that those flanking regions might carry some of the determinants of the positive correlation between sequence similarity and recombination rate. Similarly, results on A. thaliana (Ziolkowski et al. 2015) showed that the presence of a heterozygous interval next to a homozygous region leads to more COs in the heterozygous region and less in the homozygous one. At a larger scale, we observed that the genome wide correlation between recombination rate and sequence similarity is stronger than when focusing on individual chromosomes, and even more than when focusing on individual intervals. This points to the presence of trans acting factors modulating CO formation, in addition to cis effects. Further, our study of hybrid $\times$ intervals interactions indicated that the contribution of the cis effects was two times larger than that of trans effects when it comes to explaining the variance of recombination rate. Our results altogether suggest that heterozygosity alone is not sufficient to explain the variation observed in $\mathrm{CO}$ numbers and positions across hybrids, in accordance with previous works (Salomé et al. 2012; Bauer et al. 2013; Rodgers-Melnick et al. 2015; Ziolkowski and Henderson 2017). CO control may also depend on other factors such as structural differences between homologous genomes that can inhibit $\mathrm{CO}$ formation as observed in A. thaliana (Fransz et al. 2016), or modify CO frequency as suggested in maize (Bauer et al. 2013; Rodgers-Melnick et al. 2015). Beyond sequence-related effects, recombination can also be modulated by epigenetic factors, as observed in centromeric regions (N. A. Yelina et al. 2012, 2015; N. Yelina et al. 2015), and by environmental conditions (Phillips et al. 2015; Saini et al. 2017; Zhang et al. 2017).

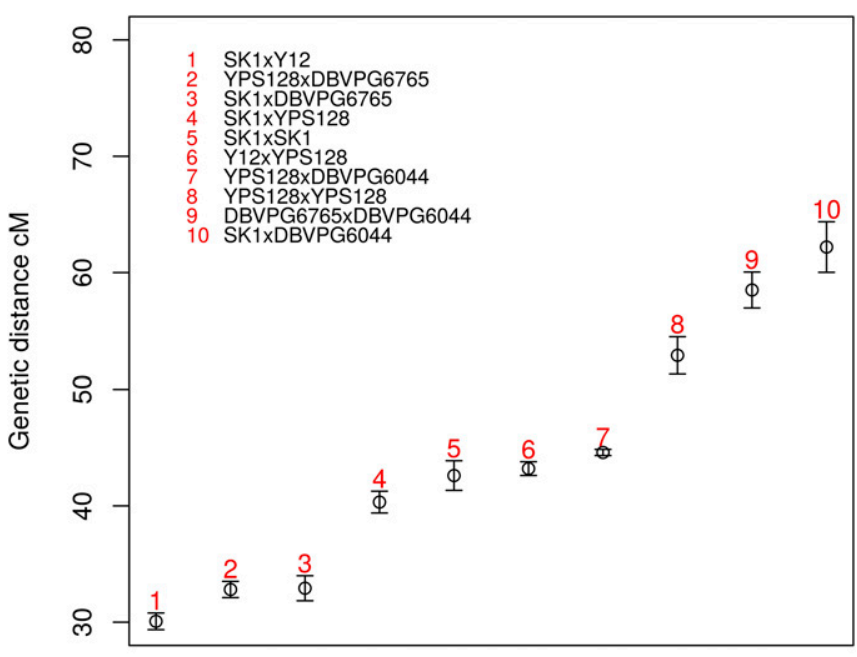

Hybrid

Figure 7 Diallel experiment: genetic length of interval R8Y9 on chromosome $\mathrm{XI}$ for 10 hybrids obtained by crossing five parental strains. Error bars indicate $95 \%$ confidence intervals.

Dissecting parental effects on recombination: Our diallel experiment also showed a significant positive effect of sequence similarity on recombination rate. Together with the correlation observed in our diversity experiment, this confirms that heterozygosity is a major determinant of the intraspecific genetic diversity of recombination rate in S. cerevisiae hybrids. Since this determinant is defined pairwise rather than in terms of individual sequences, its effect has no additive component and may be considered as overdominance. This is illustrated by comparing recombination rates of heterozygous $v s$. homozygous crosses involving the parental strains SK1 and YPS128 (Figure 6 and Figure 7): CO numbers measured in SK1 $\times$ YPS128 were significantly lower than in both SK1 $\times$ SK1 and YPS128 $\times$ YPS128 crosses, reflecting overdominance due to sequence divergence. But in fact, the quantitative analysis of the diallel experiment revealed that this apparent sequence similarity effect comes from the combined effects of $\alpha S$ and inbreeding which represent respectively the negative effect of strong heterozygosity on recombination as well as the negative effect of inbreeding in perfect homozygotes on recombination $(\alpha S=+44 \mathrm{cM}$; inbreeding effect, $-36.7 \mathrm{cM}$ for SK1 $\times$ SK1 and $-23.3 \mathrm{cM}$ for YPS128 $\times$ YPS128) (Table S4). Accordingly, the highest values of recombination in the diallel experiment do not correspond to homozygous diploids but to the heterozygous hybrid SK1 $\times$ DBVPG6044. This may be explained by the fact that SK1 and DBVPG6044 may be genetically close enough to allow high recombination rates $(\alpha S=23.35 \mathrm{cM})$ but different enough to escape inbreeding effects on recombination. It would be interesting to extend our experiment to more closely related strains to investigate in detail such inbreeding effect. It is usually assumed that inbreeding depression is due to recessive deleterious mutations (Charlesworth and Willis 2009), and this is expected to be particularly true in 
outcrossing species, which do not purge such mutations. Unfortunately, the relationship between recombination rate and fitness is complex, making interpretations difficult. In the case of $S$. cerevisiae, the HO gene can lead to mating type switch (Haber 2012) potentially favoring inbreeding which may justify the inbreeding depression we see, although the level of outcrossing in natural $S$. cerevisiae populations remains unknown (Ruderfer et al. 2006; Magwene et al. 2011).

\section{Acknowledgments}

The authors thank Christine Dillmann and Marianyela Petrizzelli for their help in analyzing the diallel experiment, as well as Valérie Borde, Monique Bolotin-Fukuhara, Denise Zickler, and two anonymous reviewers for helpful comments on the manuscript. The present work has benefited from the Cytometry/Electronic Microscopy/Light Microscopy facility of Imagerie-Gif (http://www.i2bc.paris-saclay.fr), a member of IBiSA (Infrastructures en Biologie Santé et Agronomie http://www.ibisa.net), and was supported by "FranceBioImaging" (grant ANR-10-INBS-04-01) and the LabEx "Saclay Plant Science" (https://www.universite-paris-saclay .fr/en/research/project/labex-sps-sciences-des-plantes-desaclay) (grant ANR-10-LABX-0040-SPS). Génétique Quantitative et Evolution-Le Moulon benefits from the support of the LabEx Saclay Plant Sciences-SPS (https://www.universiteparis-saclay.fr/en/research/project/labex-sps-sciences-desplantes-de-saclay) (grant ANR-10-LABX-0040-SPS).

\section{Literature Cited}

Acquaviva, L., L. Szekvolgyi, B. Dichtl, B. S. Dichtl, C. L. R. Saint Andre et al., 2012 The COMPASS subunit Spp1 links histone methylation to initiation of meiotic recombination. Science 339: 215-218. https://doi.org/10.1126/science.1225739

Aggarwal, D. D., E. Rashkovetsky, P. Michalak, I. Cohen, Y. Ronin et al., 2015 Experimental evolution of recombination and crossover interference in Drosophila caused by directional selection for stress-related traits. BMC Biol. 13: 101. https://doi.org/ 10.1186/s12915-015-0206-5

Alam, M., L. Rönnegård, and X. Shen, 2015 Fitting conditional and simultaneous autoregressive spatial models in hglm. The $\mathrm{R}$ Journal 7: 5-18.

Alani, E., R. Reenan, and R. D. Kolodner, 1994 Interaction between mismatch repair and genetic recombination in Saccharomyces cerevisiae. Genetics 137: 19-39.

Anderson, L. K., G. G. Doyle, B. Brigham, J. Carter, K. D. Hooker et al., 2003 High-resolution crossover maps for each bivalent of Zea mays using recombination nodules. Genetics 165: 849-865.

Anderson, L. K., L. D. Lohmiller, X. Tang, D. B. Hammond, L. Javernick et al., 2014 Combined fluorescent and electron microscopic imaging unveils the specific properties of two classes of meiotic crossovers. Proc. Natl. Acad. Sci. USA 111: 13415-13420. https://doi.org/10.1073/pnas.1406846111

Basu-Roy, S., F. Gauthier, L. Giraut, C. Mézard, M. Falque et al., 2013 Hot regions of noninterfering crossovers coexist with a nonuniformly interfering pathway in Arabidopsis thaliana. Genetics 195: 769-779. https://doi.org/10.1534/genetics.113. 155549
Baudat, F., J. Buard, C. Grey, A. Fledel-Alon, C. Ober et al., 2009 PRDM9 is a major determinant of meiotic recombination hotspots in humans and mice. Science 327: 836-840. https:// doi.org/10.1126/science.1183439

Bauer, E., M. Falque, H. Walter, C. Bauland, C. Camisan et al., 2013 Intraspecific variation of recombination rate in maize. Genome Biol. 14: R103. https://doi.org/10.1186/gb-2013-149-r103

Borde, V., and B. de Massy, 2013 Programmed induction of DNA double strand breaks during meiosis: setting up communication between DNA and the chromosome structure. Curr. Opin. Genet. Dev. 23: 147-155. https://doi.org/10.1016/j.gde.2012.12.002

Borts, R. H., and J. E. Haber, 1987 Meiotic recombination in yeast: alteration by multiple heterozygosities. Science 237: 1459-1465. https://doi.org/10.1126/science.2820060

Brown, M. S., E. Lim, C. Chen, K. T. Nishant, and E. Alani, 2013 Genetic analysis of mlh3 mutations reveals interactions between crossover promoting factors during meiosis in Baker's yeast. G3 (Bethesda) 3: 9-22. https://doi.org/10.1534/g3. 112.004622

Bürger, R., 1999 Evolution of genetic variability and the advantage of sex and recombination in changing environments. Genetics 153: 1055-1069.

Camacho, C., G. Coulouris, V. Avagyan, N. Ma, J. Papadopoulos et al., 2009 BLAST+: architecture and applications. BMC Bioinformatics 10: 421. https://doi.org/10.1186/1471-2105-10-421

Chakraborty, P., A. V. Pankajam, G. Lin, A. Dutta, G. N. Krishnaprasad et al., 2017 Modulating crossover frequency and interference for obligate crossovers in Saccharomyces cerevisiae meiosis. G3 (Bethesda) 7: 1511-1524. https://doi.org/10.1534/g3.117. 040071

Chakraborty, U., and E. Alani, 2016 Understanding how mismatch repair proteins participate in the repair/anti-recombination decision. FEMS Yeast Res. 16: fow071. https://doi.org/10.1093/ femsyr/fow071

Chakraborty, U., C. M. George, A. M. Lyndaker, and E. Alani, 2016 A delicate balance between repair and replication factors regulates recombination between divergent DNA sequences in Saccharomyces cerevisiae. Genetics 202: 525-540. https://doi. org/10.1534/genetics.115.184093

Chambers, S. R., N. Hunter, E. J. Louis, and R. H. Borts, 1996 The mismatch repair system reduces meiotic homeologous recombination and stimulates recombination-dependent chromosome loss. Mol. Cell. Biol. 16: 6110-6120. https://doi.org/10.1128/ MCB.16.11.6110

Charlesworth, D., and J. H. Willis, 2009 The genetics of inbreeding depression. Nat. Rev. Genet. 10: 783-796. https://doi.org/ $10.1038 / \mathrm{nrg} 2664$

Chen, S. Y., T. Tsubouchi, B. Rockmill, J. S. Sandler, D. R. Richards et al., 2008 Global analysis of the meiotic crossover landscape. Dev. Cell 15: 401-415. https://doi.org/10.1016/j.devcel.2008. 07.006

Chen, W., and S. Jinks-Robertson, 1999 The role of the mismatch repair machinery in regulating mitotic and meiotic recombination between diverged sequences in yeast. Genetics 151: 12991313.

Coop, G., X. Wen, C. Ober, J. K. Pritchard, and M. Przeworski, 2008 High-resolution mapping of crossovers reveals extensive variation in fine-scale recombination patterns among humans. Science 319: 1395-1398. https://doi.org/10.1126/science. 1151851

Cubillos, F. A., E. J. Louis, and G. Liti, 2009 Generation of a large set of genetically tractable haploid and diploid Saccharomyces strains. FEMS Yeast Res. 9: 1217-1225. https://doi.org/ 10.1111/j.1567-1364.2009.00583.x

Cubillos, F. A., E. Billi, E. Zörgö, L. Parts, P. Fargier et al., 2011 Assessing the complex architecture of polygenic traits 
in diverged yeast populations. Mol. Ecol. 20: 1401-1413. https://doi.org/10.1111/j.1365-294X.2011.05005.x

de los Santos, T., N. Hunter, C. Lee, B. Larkin, J. Loidl et al., 2003 The Mus81/Mms4 endonuclease acts independently of double-Holliday junction resolution to promote a distinct subset of crossovers during meiosis in budding yeast. Genetics 164: 81-94.

de Massy, B., 2003 Distribution of meiotic recombination sites. Trends Genet. 19: 514-522. https://doi.org/10.1016/S01689525(03)00201-4

Drouaud, J., H. Khademian, L. Giraut, V. Zanni, S. Bellalou et al., 2013 Contrasted patterns of crossover and non-crossover at Arabidopsis thaliana meiotic recombination hotspots. PLoS Genet. 9: e1003922. https://doi.org/10.1371/journal.pgen. 1003922

Dujon, B. A., and E. J. Louis, 2017 Genome diversity and evolution in the budding yeasts (Saccharomycotina). Genetics 206: 717-750. https://doi.org/10.1534/genetics.116.199216

Fernandes, J. B., M. Séguéla-Arnaud, C. Larchevêque, A. H. Lloyd, and R. Mercier, 2018 Unleashing meiotic crossovers in hybrid plants. Proc. Natl. Acad. Sci. USA 115: 2431-2436. https://doi. org/10.1073/pnas.1713078114

Fransz, P., G. Linc, C. Lee, S. A. Aflitos, J. R. Lasky et al., 2016 Molecular, genetic and evolutionary analysis of a paracentric inversion in Arabidopsis thaliana. Plant J. 88: 159-178. https://doi.org/10.1111/tpj.13262

Gerton, J. L., J. DeRisi, R. Shroff, M. Lichten, P. O. Brown et al., 2000 Global mapping of meiotic recombination hotspots and coldspots in the yeast Saccharomyces cerevisiae. Proc. Natl. Acad. Sci. USA 97: 11383-11390. https://doi.org/10.1073/ pnas.97.21.11383

Giraut, L., M. Falque, J. Drouaud, L. Pereira, O. C. Martin et al., 2011 Genome-wide crossover distribution in Arabidopsis thaliana meiosis reveals sex-specific patterns along chromosomes. PLoS Genet. 7: e1002354. https://doi.org/10.1371/journal. pgen.1002354

Haber, J. E., 2012 Mating-type genes and MAT switching in Saccharomyces cerevisiae. Genetics 191: 33-64. https://doi.org/ 10.1534/genetics.111.134577

Hunter, C. M., W. Huang, T. F. Mackay, and N. D. Singh, 2016 The genetic architecture of natural variation in recombination rate in Drosophila melanogaster. PLoS Genet. 12: e1005951. https:// doi.org/10.1371/journal.pgen.1005951

Hunter, N., 2015 Meiotic recombination: the essence of heredity. Cold Spring Harb. Perspect. Biol. 7: 1-35. https://doi.org/ 10.1101/cshperspect.a016618

Hunter, N., S. R. Chambers, E. J. Louis, and R. H. Borts, 1996 The mismatch repair system contributes to meiotic sterility in an interspecific yeast hybrid. EMBO J. 15: 1726-1733. https:// doi.org/10.1002/j.1460-2075.1996.tb00518.x

Illingworth, C. J., L. Parts, A. Bergström, G. Liti, and V. Mustonen, 2013 Inferring genome-wide recombination landscapes from advanced intercross lines: application to yeast crosses. PLoS One 8: e62266. https://doi.org/10.1371/journal.pone.0062266

Jahns, M. T., D. Vezon, A. Chambon, L. Pereira, M. Falque et al., 2014 Crossover localisation is regulated by the neddylation posttranslational regulatory pathway. PLoS Biol. 12: e1001930. https://doi.org/10.1371/journal.pbio.1001930

Kaback, D. B., V. Guacci, D. Barber, and J. W. Mahon, 1992 Chromosome size-dependent control of meiotic recombination. Science 256: 228-232. https://doi.org/10.1126/ science. 1566070

Keeney, S., C. N. Giroux, and N. Kleckner, 1997 Meiosis-specific DNA double-strand breaks are catalyzed by Spo11, a member of a widely conserved protein family. Cell 88: 375-384. https:// doi.org/10.1016/S0092-8674(00)81876-0

Kosheleva, K., and M. M. Desai, 2017 Recombination alters the dynamics of adaptation on standing variation in laboratory yeast populations. Mol. Biol. Evol. https://doi.org/10.1093/molbev/ msx278

Lam, I., and S. Keeney, 2015 Non-paradoxical evolutionary stability of the recombination initiation landscape in yeast. Science 350: 932-937. https://doi.org/10.1126/science.aad0814

Lenarcic, A. B., K. L. Svenson, G. A. Churchill, and W. Valdar, 2012 A general bayesian approach to analyzing diallel crosses of inbred strains. Genetics 190: 413-435. https://doi.org/10. 1534/genetics.111.132563

Li, C., Y. Li, Y. Shi, Y. Song, D. Zhang et al., 2016 Analysis of recombination QTLs, segregation distortion, and epistasis for fitness in maize multiple populations using ultra-high-density markers. Theor. Appl. Genet. 129: 1775-1784. https://doi. org/10.1007/s00122-016-2739-6

Liti, G., D. M. Carter, A. M. Moses, J. Warringer, L. Parts et al., 2009 Population genomics of domestic and wild yeasts. Nature 458: 337-341. https://doi.org/10.1038/nature07743

Liu, C. L., T. Kaplan, M. Kim, S. Buratowski, S. L. Schreiber et al., 2005 Single-nucleosome mapping of histone modifications in S. cerevisiae. PLoS Biol. 3: e328. https://doi.org/10.1371/journal. pbio.0030328

Liu, S., C.-T. Yeh, T. Ji, K. Ying, H. Wu et al., 2009 Mu transposon insertion sites and meiotic recombination events Co-localize with epigenetic marks for open chromatin across the maize genome. PLoS Genet. 5: e1000733. https://doi.org/10.1371/journal. pgen.1000733

Magwene, P. M., Ö. Kayıkçı, J. A. Granek, J. M. Reininga, Z. Scholl et al., 2011 Outcrossing, mitotic recombination, and lifehistory trade-offs shape genome evolution in Saccharomyces cerevisiae. Proc. Natl. Acad. Sci. USA 108: 1987-1992. https:// doi.org/10.1073/pnas.1012544108

Malkova, A., J. Swanson, M. German, J. H. McCusker, E. A. Housworth et al., 2004 Gene conversion and crossing over along the 405-kb left arm of Saccharomyces cerevisiae chromosome VII. Genetics 168: 49-63. https://doi.org/10.1534/ genetics.104.027961

Mancera, E., R. Bourgon, A. Brozzi, W. Huber, and L. M. Steinmetz, 2008 High-resolution mapping of meiotic crossovers and noncrossovers in yeast. Nature 454: 479-485. https://doi.org/10. 1038/nature07135

Marsolier-Kergoat, M.-C., M. M. Khan, J. Schott, X. Zhu, and B. Llorente, 2018 Mechanistic view and genetic control of DNA recombination during meiosis. Mol. Cell 70: 9-20.e6. https:// doi.org/10.1016/j.molcel.2018.02.032

Martini, E., R. L. Diaz, N. Hunter, and S. Keeney, 2006 Crossover homeostasis in yeast meiosis. Cell 126: 285-295. https://doi. org/10.1016/j.cell.2006.05.044

Martini, E., V. Borde, M. Legendre, S. Audic, B. Regnault et al., 2011 Genome-wide analysis of heteroduplex DNA in mismatch repair-deficient yeast cells reveals novel properties of meiotic recombination pathways. PLoS Genet. 7: e1002305. https://doi.org/10.1371/journal.pgen.1002305

Matos, J., M. G. Blanco, S. Maslen, J. M. Skehel, and S. C. West, 2011 Regulatory control of the resolution of DNA recombination intermediates during meiosis and mitosis. Cell 147: 158172. https://doi.org/10.1016/j.cell.2011.08.032

McDonald, M. J., D. P. Rice, and M. M. Desai, 2016 Sex speeds adaptation by altering the dynamics of molecular evolution. Nature 531: 233-236. https://doi.org/10.1038/nature17143

McMullen, M. D., S. Kresovich, H. S. Villeda, P. Bradbury, H. Li et al., 2009 Genetic properties of the maize nested association mapping population. Science 325: 737-740. https://doi.org/10. 1126/science. 1174320

McPeek, M. S., and T. P. Speed, 1995 Modeling interference in genetic recombination. Genetics 139: 1031-1044.

Mercier, R., C. Mézard, E. Jenczewski, N. Macaisne, and M. Grelon, 2015 The molecular biology of meiosis in plants. Annu. Rev. 
Plant Biol. 66: 297-327. https://doi.org/10.1146/annurev-arplant050213-035923

Mirouze, M., M. Lieberman-Lazarovich, R. Aversano, E. Bucher, J. Nicolet et al., 2012 Loss of DNA methylation affects the recombination landscape in Arabidopsis. Proc. Natl. Acad. Sci. USA 109: 5880-5885. https://doi.org/10.1073/pnas.1120841109

Nepoux, V., A. Babin, C. Haag, T. J. Kawecki, and A. Le Rouzic, 2015 Quantitative genetics of learning ability and resistance to stress in Drosophila melanogaster. Ecol. Evol. 5: 543-556. https://doi.org/10.1002/ece3.1379

Opperman, R., E. Emmanuel, and A. A. Levy, 2004 The effect of sequence divergence on recombination between direct repeats in Arabidopsis. Genetics 168: 2207-2215. https://doi.org/10. 1534/genetics.104.032896

Pan, J., M. Sasaki, R. Kniewel, H. Murakami, H. G. Blitzblau et al., 2011 A hierarchical combination of factors shapes the genomewide topography of yeast meiotic recombination initiation. Cell 144: 719-731. https://doi.org/10.1016/j.cell.2011.02.009

Pawlowski, W. P., I. N. Golubovskaya, and W. Z. Cande, 2003 Altered nuclear distribution of recombination protein RAD51 in maize mutants suggests the involvement of RAD51 in meiotic homology recognition. Plant Cell 15: 1807-1816. https://doi.org/10.1105/tpc.012898

Phillips, D., G. Jenkins, M. Macaulay, C. Nibau, J. Wnetrzak et al., 2015 The effect of temperature on the male and female recombination landscape of barley. New Phytol. 208: 421-429. https://doi.org/10.1111/nph.13548

Raffoux, X., M. Bourge, F. Dumas, O. C. Martin, and M. Falque, 2018 High-throughput measurement of recombination rates and genetic interference in Saccharomyces cerevisiae. Yeast 35: 431-442. https://doi.org/10.1002/yea.3315

Rodgers-Melnick, E., P. J. Bradbury, R. J. Elshire, J. C. Glaubitz, C. B. Acharya et al., 2015 Recombination in diverse maize is stable, predictable, and associated with genetic load. Proc. Natl. Acad. Sci. USA 112: 3823-3828. https://doi.org/10.1073/pnas.1413864112

Rönnegård, L., X. Shen, and M. Alam, 2010 hglm: a package for fitting hierarchical generalized linear models. The R Journal 2: $20-28$.

Ross-Ibarra, J., 2004 The evolution of recombination under domestication: a test of two hypotheses. Am. Nat. 163: 105-112. https://doi.org/10.1086/380606

Ruderfer, D. M., S. C. Pratt, H. S. Seidel, and L. Kruglyak, 2006 Population genomic analysis of outcrossing and recombination in yeast. Nat. Genet. 38: 1077-1081. https://doi.org/ $10.1038 / \mathrm{ng} 1859$

Saini, R., A. K. Singh, S. Dhanapal, T. H. Saeed, G. J. Hyde et al., 2017 Brief temperature stress during reproductive stages alters meiotic recombination and somatic mutation rates in the progeny of Arabidopsis. BMC Plant Biol. 17: 103. https://doi. org/10.1186/s12870-017-1051-1

Salomé, P. A., K. Bomblies, J. Fitz, R. E. Laitinen, N. Warthmann et al., 2012 The recombination landscape in Arabidopsis thaliana F2 populations. Heredity (Edinb) 108: 447-455. https:// doi.org/10.1038/hdy.2011.95
Serrentino, M.-E., E. Chaplais, V. Sommermeyer, and V. Borde, 2013 Differential association of the conserved SUMO ligase Zip3 with meiotic double-strand break sites reveals regional variations in the outcome of meiotic recombination. PLoS Genet. 9: e1003416. https://doi.org/10.1371/journal.pgen.1003416

Sprague, G. F., and L. A. Tatum, 1942 General vs. specific combining ability in single crosses of corn 1. Agron. J. 34: 923-932. https://doi.org/10.2134/agronj1942.00021962003400100008x

Stahl, F. W., H. M. Foss, L. S. Young, R. H. Borts, M. F. F. Abdullah et al., 2004 Does crossover interference count in Saccharomyces cerevisiae? Genetics 168: 35-48. https://doi.org/10.1534/ genetics.104.027789

Sun, X., L. Huang, T. E. Markowitz, H. G. Blitzblau, D. Chen et al., 2015 Transcription dynamically patterns the meiotic chromosomeaxis interface. eLife 4: e07424. https://doi.org/10.7554/eLife. 07424

Tham, K.-C., R. Kanaar, and J. H. G. Lebbink, 2016 Mismatch repair and homeologous recombination. DNA Repair (Amst.) 38: 75-83. https://doi.org/10.1016/j.dnarep.2015.11.010

Yelina, N., P. Diaz, C. Lambing, and I. R. Henderson, 2015 Epigenetic control of meiotic recombination in plants. Sci. China Life Sci. 58: 223-231. https://doi.org/10.1007/s11427-015-4811-x

Yelina, N. E., K. Choi, L. Chelysheva, M. Macaulay, B. de Snoo et al., 2012 Epigenetic remodeling of meiotic crossover frequency in arabidopsis thaliana DNA methyltransferase mutants. PLOS Genet. 8: e1002844. https://doi.org/10.1371/journal.pgen. 1002844

Yelina, N. E., C. Lambing, T. J. Hardcastle, X. Zhao, B. Santos et al., 2015 DNA methylation epigenetically silences crossover hot spots and controls chromosomal domains of meiotic recombination in Arabidopsis. Genes Dev. 29: 2183-2202. https://doi.org/ 10.1101/gad.270876.115

Zhang, K., X. C. Wu, D. Q. Zheng, and T. D. Petes, 2017 Effects of temperature on the meiotic recombination landscape of the yeast Saccharomyces cerevisiae. MBio 8: e02099-17. https:// doi.org/10.1128/mBio.02099-17

Zhao, H., T. P. Speed, and M. S. McPeek, 1995 Statistical analysis of crossover interference using the chi-square model. Genetics 139: 1045-1056.

Zickler, D., and N. Kleckner, 2016 A few of our favorite things: pairing, the bouquet, crossover interference and evolution of meiosis. Semin. Cell Dev. Biol. 54: 135-148. https://doi.org/ 10.1016/j.semcdb.2016.02.024

Ziolkowski, P. A., and I. R. Henderson, 2017 Interconnections between meiotic recombination and sequence polymorphism in plant genomes. New Phytol. 213: 1022-1029. https://doi. org/10.1111/nph.14265

Ziolkowski, P. A., L. E. Berchowitz, C. Lambing, N. E. Yelina, X. Zhao, et al., 2015 Juxtaposition of heterozygous and homozygous regions causes reciprocal crossover remodelling via interference during Arabidopsis meiosis. eLife 4: e03708. https:// doi.org/10.7554/eLife.03708.

Communicating editor: $K$. Bomblies 